\title{
MERCOSUL: INTÉGRATION ÉCONOMIQUE ET HARMONISATION JURIDIQUE
}

\author{
Paulo Borba Casella \\ Professor Associado do Departamento de Direito Internacional \\ da Faculdade de Direito da Universidade de São Paulo
}

\begin{abstract}
Resumo:
A integração econômica, além da simples supressão de barreiras tarifárias e do conjunto de medidas de efeito equivalente, nos obriga a enfocar o Estado e o direito sob novas perspectivas / paralelamente aos aspectos econômicos, excessivamente reiterados, devemos atentar para o alcance jurídico de qualquer processo de integração, especialmente no contexto de um 'mercado comum' / concretamente essa dimensão se coloca pela via da harmonização jurídica, como bem exemplifica o atual momento do Mercosul, completado o período de transição.
\end{abstract}

Résumé:

L'intégration économique, au delà de la simple suppression des droits de douane et de l'ensemble des mesures d'effet équivalent nous oblige à envisager l'État et le droit sous des nouvelles perspectives / en parallèle aux aspects économiques, trop souvent présentés, on doit faire attention à la portée juridique de tout processus d'intégration, notamment dans le cadre d'un 'marché commun' / concrètement cette dimension se présente par la voie de l'harmonisation juridique, comme nous en donne un bon exemple la situation présente du Mercosul, une fois completée la période de transition.

La fin de l'histoire: une thèse qui dure, une formule qui revient; de Hegel à Fukuyama. Contrairement à cette thèse, l'idée même d'Occident est celle de l'histoire qui ne s'arrête pas, qui englobe les civilisations pour lesquelles l'histoire s'est arretée. Plus qu'un simple hasard ou une nouvelle théorie, on y mettrait en cause l'Occident en tant que tel.

L'homme s'est toujours posé des questions sur soi-même et sur le monde autour de soi. Encore plus que penser, comme le voulait Descartes, se poser des questions serait le signe d'exister, l'évidence d'être là. Assez curieusement nous sommes dans une période où énormement de nouveautés se produisent, nombreuses nouvelles façons d'envisager le monde peuvent être adoptées et l'on se trouve coincée devant la fin de l'histoire. Ce qui peut se dire: se poser toujours ou bien ne 
plus se poser des questions. Or, en ce moment, il y a trop de nouveau pour être au bord de la fin de l'histoire.

Contrairement à la fin de l'histoire se dressent et le catalogue des evenements internationaux depuis l'année admirable de 1989 et des nouvelles perspectives pour envisager le monde. Point n'est besoin de reprendre un tel catalogue assez connu et à succès facile! Nous le tenons tous présent ce catalogue; il n'est point besoin de le répéter inlassablement -- autant que la répétition d'histoires connues soit la clé du succès, nous avertissait déjà sagement Ezra Pound.

Dans ce cadre changeant les dynamiques sont variables: même les données incontestables sont reprises et remises en discussion; en même temps des nouvelles vérités s'imposent du jour au lendemain. Loin de craindre la fin de l'histoire, nous devrions être ravis de pouvoir vivre un moment historique où incontestable et clairement on est en train de voir se faire l'histoire; d'autant plus qu'elle s'internationalise à un rythme inattendu; elle abandone les modèlles idéologiques pour se faire plus pragmatique; on quitte les grands slogans politiques et l'on s'occupe d'autres slogans certes, voire moins grandiloquents et en même temps l'on s'inquiète rigoureusement des taux de change, de la stabilité et de la convertibilité des monnaies, de l'équilibre des bilans de paiements, et des échanges internationaux; les droits fondamentaux de la personne, auxquels viennent s'ajouter plus récemment les droits sociaux, sont désormais des acquis communutaires, des acquis pour l'ensemble du droit international.

Tout une période de foisonnement, les perspectives peuvent changer considérablement selon le point de vue -- soit-il géographique, politique ou historique -- de l'observateur. L'inégalité est inhérente à la condition humaine. Rousseau entamait déjà en 1754 son Discours sur l'origine et les fondements de l'inégalité parmi les hommes. Il ne nous reste qu'à situer et le cadre et le contexte dans lequel se déroule le point à faire.

On peut se demander quels seraient les événements les plus marquants depuis la fin de la II Guerre mondiale; des listes divergentes pourraient être dressées, mais sûrement il y aurait lieu pour faire figurer l'intégration économique. Phénomène assez vaste, et à multiples effets, justement la comprehension de l'intégration économique peut -- c'est l'avis d'un juriste -- être située par le volet de I'harmonisation juridique.

À partir du choix du sujet, on peut situer le cadre de l'intégration économique et le contexte interdépendant de l'harmonisation juridique et dresser la 
comparaison entre l'expérience européenne et celle non pas de l'ensemble de l'Amérique latine -- un concept qui me fait déjà poser des questions en tant que tel -mais surtout du ainsi dit "Cône Sud" de celle-ci, ce qui est sufisamment vague et même temps géographiquement convenable pour être utile. Il est inévitable de vouloir établir quelques comparaisons: il reste à voir dans quelle mesure pourront être utiles telles comparaisons.

En ce moment on craint l'immobilité et l'on se pose des questions à l'intérieur de la fortéresse Europe, malgré les extraordinaires avances connues depuis 1951: depuis des années, on l'a déjà dit, oscillant entre "europhorie" et "europhobie", l'on dresse le sombre cadre d'une moins aisement maniable Europe à 15; l'on plaint une Europe à deux vitesses; l'on regrette les risques d'une Europe marche-arrière; avec raison, une Europe qui craint et vit la hantise de la reprise des nationalismes les plus durs et inhumains -- des minorités infimes, dira-t-on; malheureusement on connaît trop bien l'effet de l'action de telles minorités, tant que la majorité reste silencieuse; une Europe qui se pose des questions autour de soi-même, de son avenir, du monde autour de soi, et qui se voit concernée dans la mesure exacte où, tout comme en matière de droit de la concurrence, il y a des "effects qui se produisent sur le marché intérieur"...

Em même temps, en Amérique latine, depuis des décennies se succedaient des essais d'intégration, des variations sur un vieil air, des rebondissements autour du même sujet, sans jamais démarrer pour de bon: l'Association latino-américaine de échange (l'AELE), ensuite l'Association latinoaméricaine de développement et intégration (l'ALADI), selon les Traités de Montevideo (1960 et 1980), le Traité général centro-américain, de Managuá (1960), le Marché commun centro-américain, le Marché commun du Caribe, le Pacte andin toujours des ressemblances, on se donnait la peine de copier les formules, la façade, voire les structures sans que des résultats équivalents s'y produisent. Malgré tout, l'intégration fonctionnait en Europe, mais il n'y avait pas de progrès substantiels au Nouveau Monde.

Vers la fin des années quatre-vingt, des changements aussi considérables que dans le reste du monde semblent être déclanchés au Nouveau Monde: l'Accord de libre échange entre le Canadá et les États-Unis, de 1988, au quel vient s'ajouter le Mexique, en 1992, dans le cadre de l'Accord de libre échange nordaméricain (l'ALENA), tandis qu'au Cone Sud, après s'être historiquement méfiés l'un de l'autre, Brésil et Argentine, ayant terminé, par différentes voies, leurs respectifs 
régimes militaires, remplacent l'apparat militaire par le commerce régional: on commence à parler d'un marché commun Brésil-Argentine, on l'entâme autour de 1985, on démarre le processus en 1988, pour être rejoints par le Paraguay et l'Uruguay dans le cadre du Traité pour la constitution d'un marché commun entre la République Argentine, la République fédérative du Brésil, la République du Paraguay et la République orientale de l'Uruguay, signé à Asunción, le 26 mars 1991 (le Traité Mercosul). Ce nouveau processus est déclenché à partir de 1991, et l'on vient d'assister à la fin de la període de transition le 31 décembre 1994, cloturée par le Protocole additionnel au Traité d'Asunción sur la structure institutionnelle du Mercosul, signé à Ouro Preto, le 17 décembre 1994.

On évite la reprise de maints aspects qui avaient été adoptés auparavant pour peu de résultats concrets; on reprend le nom "Marché commun", on se laisse leurrer même par les dates, un significatif laps freudien -- le Traité d'Asunción est signé le 26 mars, mais déliberément on se dit ne pas vouloir reprendre le modèlle européen, foncer d'autres portes, tout en voulant aboutir au même endroit. Un marché commun ne se fait pas du jour au lendemain; un marché commun ne se fait pas sans structures communes; un marché commun ne se fait sans règles communes dont l'uniformité d'interprétation et d'application soit assurée par une instance juridictionnelle indépendante des tribunaux nationaux ayant juridiction sur l'ensemble du territoire integré. Ces donnés peuvent vous sembler absolumment évidentes; et pourtant, malgré la remise en question de tels principes, quelque chose est en train de se faire dans le cadre du Mercosul. Certes, il n'y a pas, au moins pour le moment et il n'y aura pas, pour les prochaines années, un "marché commun" voire même un "marché intérieur", tout comme on le connait depuis l'Europe; mais on est en train de consolider une zone de libre échange; il peut y avoir des progrès, à partir de 2001, sous forme d'une union douanière, pour le moment imparfaite, mais en train de progresser, vers l'intérieur, dans la mesure où il y a des exceptions qui se reduisent, dans les quatre années qui suivent, entre l'Argentine et le Brésil, dans les cinq pour le Paraguay et l'Uruguay, et aussi vers l'extérieur, par un tarif externe commun (TEC) et un code douanier commun, depuis de ler janvier 1995; en même temps on est en train de négotier, d'élargir le cadre pour accueillir le Chili et la Bolivie, par des accords préalables jusqu'au 30 juin 1995, on vient de le dire lors du dernier sommet à Ouro Preto, le 16-17 décembre 1994.

L'étendue de l'intégration économique et d'un certain dégré d'harmonisation juridique qui sont en train de se faire dans le cadre du MERCOSUL 
dépassent les exigences d'une simple zone de libre échange voire même d'une union douanière, sans se présenter encore sous forme d'un marché commun. Assez dur à classer, vous me direz; autant dirait-on encore aujourd'hui à propos de l'Union européenne, où les éléments d'intégration et de coopération se combinent en dehors des formules pré-établies, où l'on a fait des progrès extraordinaires -- dont je suis enthousiaste et me suis porté le semeur, ayant publié le premier livre sur le droit communautaire au Brésil -- tout en se demandant qu'est-ce qui va se passer en 1996 et au-delà.

Est-ce que les rôles se renversent? Pas du tout. Mais, on est en train de le voir: les processus d'intégration ont une dynamique qui leur est propre; cela ne s'improvise pas; cela change difficillement; pour de bons ou pour des mauvais tours ... mais néanmoins peut-on avoir des différentes voies pour aboutir aux mêmes endroits?

Dans une telle mesure il peut avoir un sens de venir ici vous adresser la parole aujourd'hui à propos du Mercosul, voire de l'expérience interaméricaine -je ne trouve mal à l'aise avec cette abstraction dépourvue de contenu dont on se sert d'autant plus facilement que moins on songe à ce que peut bien être une "Amérique latine"; vue de l'intérieur, les différences s'imposent au-delà des ressemblances. "Amérique latine" n'a de sens que par rapport à cette autre Amérique non-latine, mais il n'y a pas forcément d'opposition entre les deux. L'usage est courant, il le restera probablement, tout comme on parle d'Amérique, la terre d'Américo Vespuccio, parce qu'il l'a décrite, il a su la rendre visible et attrayante pour les Européens, qui ne se sont pas souciés de ne pas lui donner le nom de celui qui l'avait découverte. Au moins, il y a-t-il un pays qui le souvient, la Colombie.

Les différences entre le Brésil et l'ensemble de cette Amérique qu'après mon caveat je me résigne à appeller "latine" peuvent être multiplement illustrés: on ne s'est pas tellement soucié de Colombo au Brésil; en revanche Portugal et Brésil vont fêter 500 ans de la découverte en avril 2000.

Le sens, le but de venir ici, dans ce cadre prestigieux et accueillant, vous présenter quelques aspects de l'intégration économique dans le cadre du Mercosul, de bien vouloir susciter des discussions autour du sujet de l'intégration économique, par le volet de l'harmonisation juridique, c'est de pouvoir faire des comparasions. Là peut-on bien apprendre quelque chose, remarquait Michel de Montaigne: "se frotter la cervelle d'autrui". Eh bien, nous y sommes. 
Justement je tiens à me voir dans une situation où la tâche devient plus facile à décerner, dans la mesure où l'on choisit un aspect où se manifeste l'étendue et la dimension de l'intégration, ou bien l'harmonisation dirait-on, justement où se combinent les éléments économique et juridique.

L'harmonisation juridique, dans un contexte d'intégration économique se présente simultanément comme un outil et comme un but. On la mentionne déjà dans le Traité de Rome de 1957, en parallèle avec l'uniformisation et l'harmonisation, articles 100 à 102, avec les modifications apportées par le Tue. On en fait de même dans l'article premier, alinea 4, du Traité d'Asunción de 1991, verbis: "l'engagement pour les États-parties d'harmoniser ses législations, dans les domaines concernés, pour renforcer le processus d'intégration"

L'harmonisation juridique non seulement est simultanée et peut contribuer pour renforcer l'intégration économique mais elle est la base pour faire progresser l'ensemble de l'intégration. Comme prévoit le Traité d'Asunción de 1991, dans son art. 3, "au cours de la període de transition, qui se déroulera de l'entrée en vigueur du présent Traité jusqu'au 31 decémbre 1994, et pour faciliter la constitution du marché commun, les États-parties adoptent un Régime général d'origine, un Système de règlement de différends et clauses de sauvegarde, qui constituent les Annexes II, III et IV" au Traité de 1991. Ce qui est assez surprenant, c'est de constater qu'on l'a effectivement fait dans les délais prévus: le Tec, le régime temporaire des exceptions à celle-ci, le régime d'origine Mercosul, le régime applicable aux zones franches et zones douanières spéciales (Décs. CMC ns. 5, 6, 7 et $8 / 94)$.

Au sens le plus large, ce sont des matières indispensable pour établir un dégré zéro de l'intégration économique, mais, en même temps, c'est aussi bien de l'harmonisation juridique. L'harmonisation ne se présente pas comme un but absolu ou comme une fin en soi, mais plutôt ce serait un outil pour assurer le dégré indispensable d'approximation entre dispositions légales, règlementaires et administratives des États-membres, pour les matières, "ayant une portée directe sur l'établissement ou le fonctionnement du marché commun", le prévoyait déjà la rédaction originelle de l'article 100 du Traité de Rome de 1957, d'où découlerait que l'harmonisation serait, au moins en principe, limitée aux matières où la diversité des dispositions nationales pourrait entraîner des obstacles au bon fonctionnement du marché commun. 
Dans la pratique, une telle cesure s'est averée excessivement conservatrice et restreinte, dans la mesure où plusieurs domaines, e.g. en matière d'harmonisation fiscale, on est allé bien au-délà d'une telle configuration, vues les exigences du processus d'intégration économique et les conséquences qui se sont manifestées progréssivement. Des nouveaux domains entiers ont été inclus dans le cadre communautaire tels la protection aux consommateurs, le droit de l'environnement et autres.

Or, il n'est point besoin de reprendre des questions concernant l'harmonisation des législations des États-membres de l'Union européenne, mais de cerner son équivalent dans de cadre du Mercosul. Le parallèle se laisse dresser.

La question de l'harmonisation dans le cadre du Mercosul se pose assez souplement: prévue depuis l'article premier du Traité de 1991, elle reste à se faire. Il y a déjà tout un corpus juris communis qui se développe, dont aussi des textes concernant l'harmonisation. Parmi des dizaines de Résolutions et Décisions on peut citer: des plans trienaux coordonés en matière d'education (Déc. CMC 7/92) la la reconnaissance mutuelle des diplômes et des certificats de niveau élémentaire et niveau moyen non-technique (Déc. CMC 4/94); de la règlementation minimale des marchés de capitaux (Déc. CMC 8/93) à l'harmonisation des subventions aux exportations (Déc. CMC 10/94).

Le foisonnement de la règlementation existe, mais il n'est pas le seul aspect. Dans ce sens il faut surtout considérer les effets externes d'une telle coordination ou harmonisation, telles la coordination des quatre pays du Mercosul vis-à-vis d'autres pays de l'ALADI et des pays tiers (i.a., Résolutions GMC nn. 22/93 et 39/93), la possibilité d'une zone de libre échange entre l'Union européene et le Mercosul -- dans le cadre de l'Accord interregional signé à Madrid les 15 et 20 décembre 1995 -- à condition que celui-ci ne soit pas dépourvu de sens et vidé e'effets par le changement du SGP -- aussi bien que la possibilité assez incertaine mais qu'on ne pourrait pas négliger, d'une zone continentale américaine de libre échange, l'ALEA l'Association de libre échange américaine, qui reste d'ailleurs un vrai aléa pour le moment.

Il y a un processus d'harmonisation en cours, dans le cadre d'un processus d'integration économique auxquels s'ajoutent des efforts de coordination d'action vers l'extérieur qui sont en train de se faire dans le Mercosul. Ce qui montre qu'il y a quelque chose qui est en train de se faire. 
Beaucoup plus intéressant que dresser l'inventaire de toute la législation du Mercosul qui pourrait aussi avoir une portée à propos de l'harmonisation, depuis les emballages jusqu'aux etiquettes, des sauces mayonnaises et d'autres sauces jusqu'aux residus de monomère de chlore, qui serait assez efficace pour vous faire endormir, puisque vous connaissez une histoire pareille, et cela depuis quarante ans, se présentent simultanément des phénomènes d'harmonisation qui sont en train de se faire en dehors sinon à coté du Mercosul, aussi bien dans un cadre multilatéral interaméricain que dans des cadres bilatéraux.

Ces phénomènes d'harmonisation sont d'autant plus intéressants que ses résultats sont déjà présents, en plusieurs domaines: en matière de droit international, depuis 1975, par la suite 1979, 1984, 1989 et 1994, sous forme de plus d'une vingtaine de Conventions interaméricaines de droit international privé (les CIDIP's). Celles-ci ne sont pas notre affaire aujourd'hui, mais présentent un intérêt par elles-mêmes, dans la mesure où il y a déjà de résultats qui se sont produits et qui sont en train de se produire dans des délais très courts; aussi bien par le volet bilatéral en plusieurs domaines, dont des matières cruciales comme le droit des sociétés et le droit fiscal, par le Traité entre le Brésil et l'Argentine instituant le régime des sociétés binationales; et en matière fiscale, la Convention entre le Brésil et l'Argentine pour éviter la double imposition et l'évasion fiscale.

Les cinq conférences interaméricaines de droit international privé, les CIDIP, se sont tenues successivement en 1975 au Panamá, en 1979 à Montevideo, en 1984 à La Paz, en 1989 encore à Montevideo -- pour fêter le centennaire des Traités de Montevideo de 1889 -- et dernierement en 1994 au Mexique. Une sixième doit se tenir dans un délai des quatre ou cinq années qui suivent. Des quatre premières CIDIP resulterent 21 Conventions, et 1a 5e. CIDIP, Mexique, 1994, mérite mention à part par la récent et très importante Convention interaméricaine sur la loi applicable aux contrats internationaux.

La majorité des CIDIP's a été déjà signée par les quatre États-membres du Mercosul, dont la moitié est en vigueur, la plupart ayant déjà reçu les ratifications du Paraguay et de l'Uruguay. L'Argentine à son tour, a déjà ratifiée une dizaine des CIDIP's. Le Brésil avait signée la plupart des Conventions mais ne les avait pas encore ratifiées. Maintenant une quinzaine de ces ratifications sont en cours dans le Congrès brésilien. Deux ont été ratifiées en 1994 suivies de plusieurs autres dans le courant de l'année 1995. Dans un bref délai ces Conventions pourront aboutir à 
l'uniformisation des règles de conflit pour l'ensemble du Mercosul, ce qui est un progrès considérable.

La liste des CIDIP comprend quatre grands domaines: les règles de droit international privé, le droit commercial et l'arbitrage, le droit de la procédure civile et le droit de famille. A partir de ces quatre grandes branches, les Conventions se ramifient, depuis les règles générales de droit international privé, le domicile des personnes physiques, la personnalité et la capacité des personnes physiques en droit international privé, les conflits de lois en matière de lettres d'échange et factures, le chèque, l'arbitrage commercial, les conflits de lois en matère de personnes morales commerciales, les règles de conflit en matière de transport international de marchandises, les commissions rogatoires, l'obtention de preuves à l'étranger, le régime légal des pouvoirs pour être utilisés à l'étranger, l'éxecution de mesures intérimaires, la preuve et l'information sur le droit étranger, la compétence internationale pour l'efficacité extraterritoriale des sentences étrangères, l'efficacité extraterritoriale des sentences arbitrales étrangères, les conflits de lois en matère d'adoption internationale de mineurs, la convention sur la restitution internationale de mineurs, la convention sur les aspects civils et pénaux du trafic international de mineurs.

La Convention interaméricaine sur la loi applicable aux obligations contractuelles, de 1994, qui n'est pas encore en viguer, aura un rôle à jouer. On la compare à la Convention de Rome de 1980, et dans une certaine mesure aussi à la Convention de Vienne sur la vente internationale de marchandises. En ce domaine il y a aussi un Protocole dans le cadre du Mercosul en matière de juridiction internationale. Il faudra coordoner ces textes pour réussir à les faire fonctionner.

Bref, il y a deux mouvements qui sont décernables: d'un coté, un processus d'intégration économique a sa vis attractiva, il entraîne l'harmonisation pour "l'ensemble des matières ayant portée directe sur l'établissment et le fonctionnment du marché commun" et même au-delà -- il est surtout intéressant d'examiner les matières qui ne sont pas directement concernées par un marché commun, où l'harmonisation se fait de même; simultanément, il y a un mouvement à l'échelle continentale que l'on pourrait comparer à l'activité de la conférence de La Haye en vue de l'uniformisation du droit international privé interaméricain, qui s'exprime par l'activité des CIDIP's et qui aboutit, exactement en ce moment, -surtout depuis un point de vue brésilien --, à des résultats répérables. 
Une évaluation de la situation de l'harmonisation des législations des États-membres du Mercosul nous fait constater une remarque qui avait déjà été avancée préalablement: on dépasse le cadre d'une zone de libre échange, voire d'une union douanière, sans pour autant être situés dans un marché commun qui soit présent dans tous ses éléments. Il y a des éléments qui peuvent y mener, sans pour autant avoir déjà manifesté toutes les exigences techniques et institutionnelles dont on en aura besoin.

En même temps il est extrêmement positif de voir quelques développments récents au Brésil: on n'a pas su profiter de la révision constitutionnelle de 1994 pour frayer la voie pour l'intégration; cela reste à faire et il faudra le faire. Ceci doit être repris par le gouvernement Cardoso à partir du 15 février 1995, par tranches successives. En même temps on est en train de reformuler la loi brésilienne sur les conflits de lois, pour y admettre, i.a., une plus souple règlementation de l'autonomie des parties en matière de loi applicable aux contrats internationaux en même temps qu'une plus efficace et simple reconnaissance des sentences arbitrales étrangères.

Il y a beaucoup à faire, mais on ne peut néglier des développements extrêment intéressants qui se dressent et non plus ce qui se fait soit dans ce cadre, soit en dehors ou à côté du cadre du Mercosul en matière d'intégration économique et d'harmonisation législative. Dans ce sens, je le disais au début, on pourrait le reprendre, en paraphrasant Brillat-Savarin, loin de vouloir épuiser l'histoire, on doit n'en prende que la fleur.

Je suis à votre disposition pour donner suite à notre entretien par des questions et vous en suis reconnaissant de l'attention.

Notes concernant le texte

La "Convenção destinada a evitar a dupla tributação e prevenir a evasão fiscal em matéria de impostos sobre a renda", signée entre la République Fédérative du Brésil et la République Argentine, à Buenos Aires, le 17 mai 1980 (au Brésil: dec.-leg. n. 74, du 5 décembre 1981; en vigueur, le 7 décembre 1982; promulguée par le décret n. 87.976, du 22 décembre 1982; textes d'application de cette convention sont la "portaria" n. 22/83 (du 20 jan. 1983); D.O. 25 jan. 1983) et l'"Ato Declaratório Normativo" du CST n. 06/90 (du 20 jun. 1990) qui 
spécifie le régime applicable aux entreprises étrangères de transport (v. P.B. Casella (org.), Direito internacional tributário brasileiro, S. Paulo, LTr Ed., 1995; esp. ch. IV: Argentina, passim).

Pour une approche historique de l'évolution du DIP dans l'ensemble du continent américain, à propos v., de Jürgen Samtleben, son remarquable Internationales Privatrecht in Lateinamerika: Der Código Bustamente in Theorie und Praxis (Tübingen, Mohr Verlag, Band I: Allgemeiner Teil, 1979); aussi bien son "A codificação interamericana do Direito Internacional Privado e o Brasil" (in P.B. Casella e N. Araújo (coord.), Integração jurídica interamericana: as Convenções interamericanas de direito internacional privado, $\mathrm{S}$. Paulo/Rio, en prép. 1996). 